Calorimetry at $0^{\circ}$ in RHIC: Some Issues in the Choice of Detector Technology

\author{
Bill Christie, Sebastian White \\ Brookhaven National Laboratory \\ Upton, N.Y. 11973 \\ Philippe Gorodetzky \\ CERN, Geneva, Switzerland \\ Dragoslav Lazic \\ Florida State Univ.
}

\title{
MASTER
}




\title{
Calorimetry at $0^{\circ}$ in RHIC: Some issues in the Choice of Detector technology
}

\author{
Bill Christie and Sebastian White \\ Brookhaven Lab \\ Phillipe Gorodetzky \\ CERN \\ Dragoslav Lazic \\ Florida State University
}

We discuss briefly the case for a zero degree calorimeter at RHIC and space constraints on such a device. Two GEANT simulations of quartz fiber calorimeters are then studied and compared to the corresponding case of scintillator sampling.

We find that the $Q$-cal calorimeter does provide some improvement in response uniformity near the calorimeter edge to hadronic showers (due to the filtering out of wide angle shower products). This may be an advantage when space is limited and the calorimeter transverse dimensions are small.

\section{Motivation: the role of $0^{\circ}$ Calorimetry in event characterization.}

When two ions collide, they deposit a certain energy that is used in one or both of two ways: to make classical nuclear hadronic matter (HAD) or a plasma of quarks and gluons (QGP). An important part of the game is to distinguish between these two processes, realizing that QGP formation may be very small compared to HAD. There are 3 main tools to measure the violence of the reaction:

a) Transverse energy, $E_{T}$, calorimeter, generally an EM cal detecting the $\pi^{0}$ flux;

b) Multiplicity, $\langle M\rangle$, detector;

c) Zero degree calorimeter (ZDC), a hadron calorimeter in which the beam spectator nucleons deposit their energy.

We can divide the experiments in two kinds: fixed target, all of them up to now, and collider: our concern here.

Fixed target: All fixed target experiments had an $\mathrm{E}_{T}$ and a multiplicity measurement. Some of them, generally the ones with a loose trigger (low luminosity), had also a ZDC. All experiments show a good proportionality between the 3 measurements. This is completely consistent with the non-observation of QGP, or rather, only the observation of classical hadronic physics if the number of QGP events is very small compared to HAD ones. This is certainly true in the loose trigger experiments and could not be verified in the tight trigger expt. NA38 (muon pairs) because they had no ZDC. They were relying only on $E_{T}$ and the extraction of the number of participating nucleons is very model dependent (contrary to the case of a ZDC). The relation between $\mathrm{E}_{T}$ and multiplicity may be very different for QGP events (increased $\mathrm{p}_{T}$ ) than for HAD events. This actually means that, even if NA38 had a bigger ratio of QGP to HAD events, 
maybe they were not looking at the right bin in $\mathrm{E}_{T}$. This is why it is proposed by NA50 to use a ZDC which works best if the beam and the target are identical, e.g. $\mathrm{Pb}$ on $\mathrm{Pb}$.

A good scenario to detect a QGP would be to do an experiment with identical heavy ions and a tight trigger (strange particles, muon pairs -that are not suppressed-, etc.) and measure the longitudinal energy spectrum (related directly to $\mathrm{E}_{Z D C}$ ). Then for each bin in that spectrum, look at the correlation between $\mathrm{E}_{T}$ and the mean multiplicity $(<M>)$ to see if it deviates from the HAD one.

Collider: Now, let us come to the collider problem: here it is impossible to detect all the spectator fragments with ZDCs because it would block the beams. But we can select some of the fragments taking advantage of the magnets which bend the beams around the interaction region. The neutrons (100 GeV in RHIC's case) go straight and can be detected in a calorimeter located inside the aperture of the first magnet. The protons are bent twice as much as the $Z=A / 2$ fragments, and so, roughly, a little more than twice the beam. They could be detected in a calorimeter placed to the side of the beam tube transporting the deflected beams.

The problem is the non-detection of all the energy carried by fragments different from free neutrons and protons. To extrapolate to the full longitudinal energy is model dependent and that could ruin the demonstration made in the clean fixed target case. For instance, if only a few neutrons are detected, it can classically mean that the impact parameter was very small, or, that the impact parameter was very big, leading to the forward emission of an unseen big fragment that emits a few neutrons! However, if the protons are detected, this problem could be alleviated. This problem has to be studied in more detail, through more elaborate Monte-Carlo simulations and two measurements should be performed this year at BNL with a $\mathrm{Au}$ beam, and CERN with a $\mathrm{Pb}$ beam to count how many free neutron and proton beam spectators are emitted in correlation with the full longitudinal energy.

If this correlation is narrow enough, then $\mathrm{ZDCs}$ for neutrons and protons could be used in RHIC detectors (or ALICE at CERN) as part of a trigger. Even if this correlation is not very narrow, the ZDC could still have a useful role in RHIC experiments. For example, it may provide a clean and stable luminosity monitor. In that case, only measuring the free neutrons and forgetting the protons is probably enough.

\section{Some issues in the choice of calorimeter technology}

Why consider quartz fiber calorimetry (Q-Cal) for these tasks? There are many reasons.

First and most important, the response seen by Q-Cal to a hadronic shower is expected to be very narrow: This is due to the different type of sampling used by Q-Cal where, because of the Cherenkov effect, the main filter is that only the particles going through the fibers within a narrow angular range are detected.

A narrow shower is essential in our case for several reasons: There are only 15 $20 \mathrm{~cm}$ free in the magnet where the neutrons will be detected, and, also, the fiducial volume is big, which means we can detect the protons with a $100 \%$ efficiency quite close to the beam pipe.

Second, it is radiation resistant to a Gigarad level. 
Third, quite important also is the immunity to background, be it particles whose effect is the creation of a charged particle with a $\beta$ lower than the Cherenkov threshold, or particles along the beam line that would not be seen because they have to originate from the interaction region to be in the appropriate angular range for the Cherenkov photons to be collected.

Finally, the Cherenkov effect is fast, which is a bonus when used in a trigger.

\section{The opportunity for a 0 degree detector}

Figure 1 shows a possible beam tube geometry for a typical interaction region at RHIC. The splitting dipole (DX) geometry and the choice of a room temperature beam tube for the region between DX and the next beam element (D0) makes it possible to consider instrumenting the region at 0 degrees and just outside the beamtube.

In what follows we calculate the available space which could be instrumented from $\mathrm{z} \sim 18.2$ to 21.5 meters and between the beams.

There are two possible crossing geometries that will ever be considered at RHIC:

-1) Equal species running. In this case the beams collide at 0 degrees and are subsequently bent through $18.86 \mathrm{mrad}$ in the DX magnet.

-2) Unequal species running. In the extreme case of p-Au collisions, the beams again collide at 0 degrees, however their collision axis is tilted by $3.82 \mathrm{mrad}$ relative to the line of symmetry between the two rings.

In the Dx magnet, the proton beam is bent by $23.0419+3.823-26.865 \mathrm{mrads}$ and crosses over the symmetry line, while the $\mathrm{Au}$ beam is bent by only 23.04193.823-19.219 mrads. Preventing proton beam scraping while running p-Au collisions will determine the maximum transverse extent of a zero degree detector placed between the beamtubes.

\section{Beam trajectories:}

We will calculate the beam orbit location and transverse size at a $\mathrm{z}$ location of $18.2 \mathrm{~m}$ from the interaction point, which is somewhat arbitrarily chosen as a reasonable location for the front face of a calorimeter.

The trajectories are derived from an internal note of Steve Tepikian. A few relevant parameters are reproduced from his "TableIII. Crossing Point Geometry" in Table I. below.

Table I. Beam trajectories after DX dipole from S.Tepikian

\begin{tabular}{cccc} 
running mode & beam & $\Theta(\mathrm{mrad})$ & $\mathrm{h}(\mathrm{cm}) @ \mathrm{z}=20.5 \mathrm{~m}$ \\
\hline $\mathrm{p}-\mathrm{Au}$ & $\mathrm{p}$ & 23.042 & 15.94 \\
p-Au & $\mathrm{Au}$ & 14.679 & 17.446 \\
p-p or Au-Au & either & 18.86 & 16.694
\end{tabular}

The transverse location of the beam orbit at any $z$ location (here we take $z=18.2 \mathrm{~m}$ ) in centimeters is then: 


$$
\mathrm{h}(18.2 \mathrm{~m})=\mathrm{h}(20.5)-\Theta \times 100 . \times(20.5-18.2)
$$

which yields $\mathrm{h}=10.65,14.07$ and $12.36 \mathrm{~cm}$ for the 3 cases listed in the above table, respectively.

\section{Computing the beam envelope:}

We will use as a rule of thumb that the nearest obstruction should not come nearer than $6 \sigma$ from the beam trajectory in any optics from acceleration through late in a store (when the beam emittance, $\varepsilon$, reaches its maximum value). The beam $\sigma$ value will depend on the local betatron amplitude, the beam energy $\left(=\mathrm{\gamma mc}^{2}\right)$ and the emittance according to:

$$
\sigma^{2}=\left(\varepsilon \beta_{18.2} /(6 \pi \gamma)\right.
$$

Since there are no focusing elements between $18.2 \mathrm{~m}$ and the interaction point, the local $\beta$ amplitude is given by :

$$
\beta_{18.2}=\beta^{*}+(18.2 m)^{2} / \beta^{*}
$$

and the largest beam size will be obtained when $\beta=1 \mathrm{~m}$, and $\beta_{18.2}=332 \mathrm{~m}$.

Here we consider a normalized emittance of $40 \pi \mathrm{mm}$ mrad, which is probably too pessimistic for proton beams late in a store. We then obtain

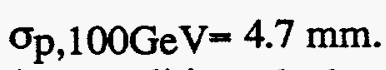

while for injection conditions the beam will be smaller. 


\section{-DX-DO BEAM TUBE CONCEPT-}

TD MINIMIZE MANUFACTURING COMPLEXITY AND PRQDUCTION COSTS BEAM LINE GEOMETRY FROM TABLE III. CRDSSING POINT GEDMETRY TEPEKIAN $43 / 92$ IR
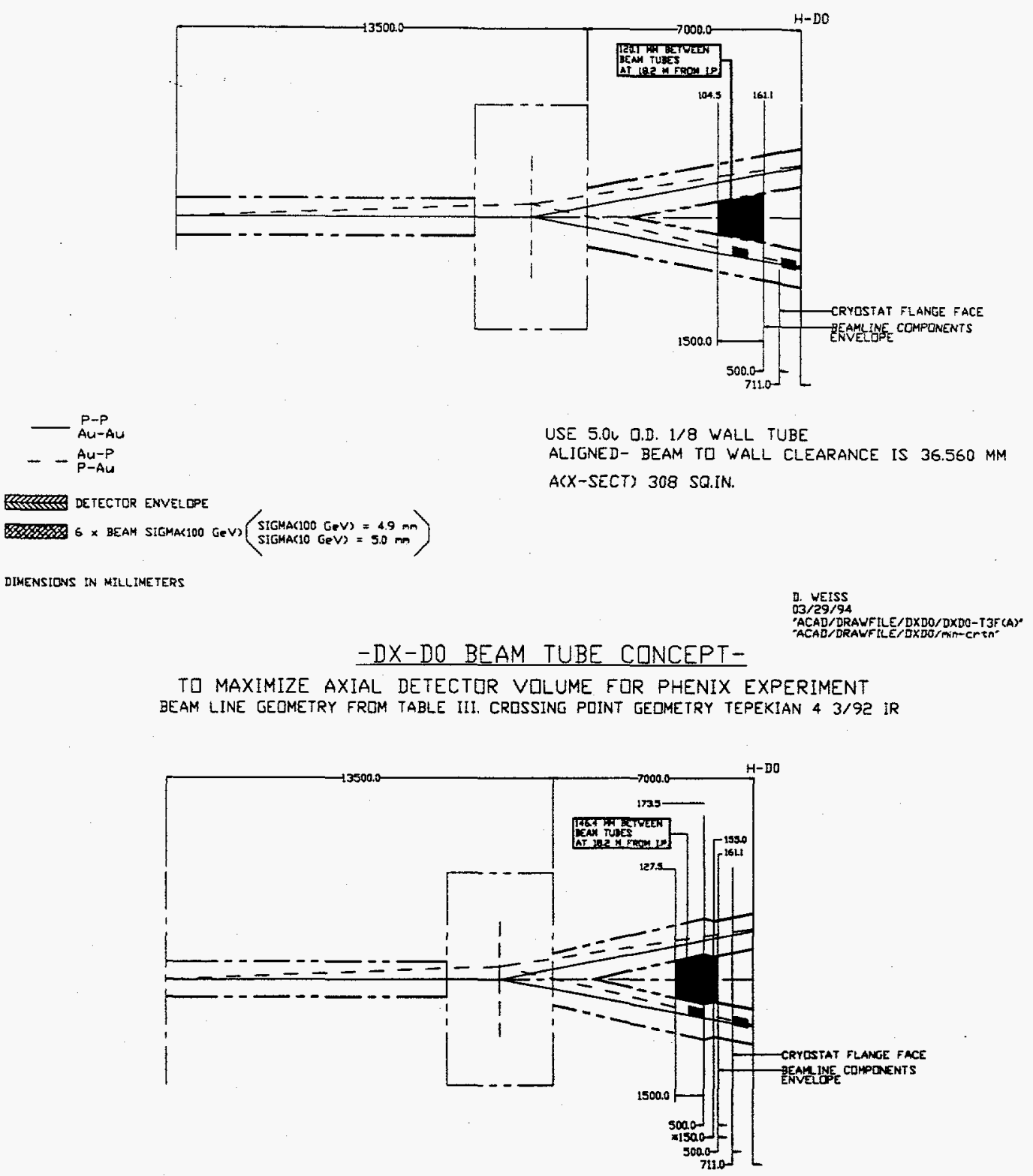

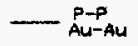

- Au-P

DETECTDR EMVELOPE

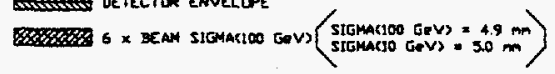

DINENSIDNS IN MILLIMETERS
* I5 CM ALLUWANCE FUR CLRRECTIDN TO NUMINAL BEAM TUBE INSTALlation USE 5.00 D.D. 1/8 WALL TUBE ALIGNED- BEAM TO WALL CLEARANCE IS $36.560 \mathrm{MM}$ MINIMUM- BEAM E TO WALL CLEARANCE IS $30.00 \mathrm{MM}$ $A(X-S E C T) 359$ SQ.IN.

Figure 1. Beam trajectories and 60 envelope (hatched box) for like species (solid lines) and poAu (dashed. lines) colliding beams. The standard beamtube arrangement (top figure) would allow for a detector footprint with typical transverse dimensions of $12 \mathrm{~cm}$. A slightly modified version (bottom figure) results in a free space of $15 \mathrm{~cm}$. 
The proposed beam tube for this region of the accelerator is $5 "$ o.d., $1 / 8$ " wall thickness steel tubing, so a beam tube which is placed so as to lie outside the worst case beam envelope will be compatible with a detector which has transverse size of

$$
h_{\text {detector }} 2 *\left(10.65 \mathrm{~cm} .-6 * 0.47 \mathrm{~cm}-1 / 8^{\prime \prime}\right)=15.06 \mathrm{~cm} \text {. }
$$

with a reasonable coverage need only have this transverse size in the plane of the accelerator where the beamtubes make their closest approach. Figure 2 shows the situation in terms a of a cut through the beams at $\mathrm{z}=18.2$ meters. It should be noted that with this beamtube arrangement, one could also instrument "outside" the beam forward direction to within about 2-1/2" from the beam. This is the region which would center line of machine

Figure 2. Cut through the Beamtube at $z=18.2 \mathrm{~m}$ from the interaction region. A calorimeter at $0^{\circ}$ must have a total transverse extent of $15 \mathrm{~cm}$ or less in the plane of the beams. intercept forward going, lower rigidity nuclear fragments.

\section{Monte Carlo studies}

Elaborate detector simulation studies of the Q-cal concept were carried out and documented in the Thesis of Dragoslav Lazic ${ }^{1}$. To get first hand knowledge of how the Q-cal works, we carried out a simplified set of simulations. The primary differences between how a standard lead sampling calorimeter and a Q-cal type calorimeter work are the velocity threshold for the Q-cal $(\beta \geq 1 / n, n=$ index of refraction) and the angular dependence imposed by the requirement that one get total internal reflection for the Cherenkov photons in the quartz fibers. 


\subsection{Simulations}

We studied the response of a lead - plastic scintillator sampling calorimeter using the detector simulation code GEANT. The coordinate system used in the simulation had the incoming particles along the positive $Z$ axis. The geometry simulated consisted of a rectangular lead block, with dimensions of $40 \mathrm{~cm}$ in the $X$ and $\mathrm{Y}$ directions and a length (along $Z$ ) of $3.0 \mathrm{~m}$. Six hundred square sheets of plastic were placed in the lead block, with dimensions of $40 \mathrm{~cm}$ by $40 \mathrm{~cm}$ in the $X$ and $Y$ directions and 1 $\mathrm{mm}$ in length along the beam, with centers along the beam every $5 \mathrm{~mm}$. The final geometry thus had a volume ratio of lead to plastic of four to one. For each particle that crossed one of these plastic layers the following information was recorded:
1.) $X$
2.) $\mathrm{Y}$
3.) $\mathrm{Z}$
4.) $\mathrm{Px}$
5.) $\mathrm{Py}$
6.) $\mathrm{Pz}$
7.) $\mathrm{P}$
8.) $\mathrm{dE} / \mathrm{dx}$
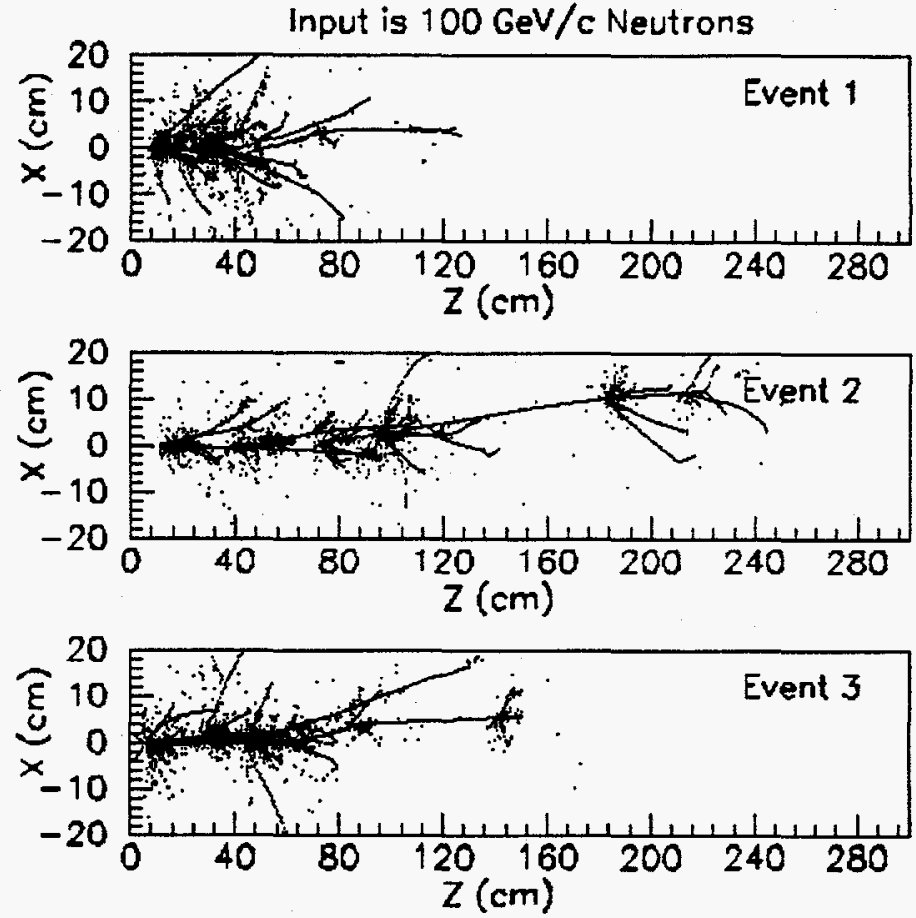

Figure 3. Top view of the charged particle "hits" in the plastic layers for three events. Input for each event is a neutron with a momentum of $100 \mathrm{GeV} / \mathrm{c}$. 
High $\mathrm{E}$ cuts $\equiv 10 \mathrm{MeV}$ for Hadrons, $1 \mathrm{MeV}$ for $\gamma s$, and $1 \mathrm{MeV}$ for $\mathrm{e}^{ \pm}$.

Low $\mathrm{E}$ cuts $\equiv 10 \mathrm{MeV}$ for Hadrons, $500 \mathrm{keV}$ for $\gamma s$, and $150 \mathrm{keV}$ for $\mathrm{e}^{ \pm}$.

Fig. 3 shows a top view of the charged particle hits in the plastic layers for three 100 $\mathrm{GeV} / \mathrm{c}$ neutron events.

Analysis

The analysis was all done using PAW. A basic assumption used in the analysis was that the number of charged particle hits in the plastic layers of the calorimeter was directly proportional to the total energy deposited in the plastic layers. For example, to get the energy resolution for a given calorimeter configuration and hit criteria we would run 50 events with a $100 \mathrm{GeV} / \mathrm{c}$ neutron incident on the calorimeter, plot out the distribution and rms for the number of hits/event, and the "simulated" percentage energy resolution would be:

$$
\text { Energy Res. }(\%)=\frac{r m s(\# \text { of hits dist. })}{\text { mean }(\# \text { of hits dist. })} * 100 \%
$$

The validity of using just the number of charged particle "hits" in the plastic layers, as opposed to summing up the energy lost by the particles as they traverse the plastic, is due to the large number of hits for each event. A single $100 \mathrm{GeV}$ incident neutron results, on average, in $\sim 890$ hits (with the Q-cal $\beta$ and $\theta$ cuts), and the Landau fluctuations applicable for each individual hit average out.

In the analysis we used the same simulated data sets to investigate the Energy resolutions of both a standard lead - scintillator sampling calorimeter and a Q-cal type calorimeter. One simply places criteria (cuts) on the hits which are included in the sums. For example, for a standard calorimeter one uses all of the charged particle hits, while for a Q-cal calorimeter one only includes hits which are due to charged particles with $\beta \geq 0.7$ and angles $\leq 10$ degrees relative to the normal of the plastic sheets (N.B. This angle cut comes from the work of D. Lazic).

To investigate the effect of finite transverse dimensions on the energy resolutions of both types of calorimeter, cuts were applied such that only the hits within a rectangular box centered on the incident beam particle were included in the sums. For example, to get the energy resolution for a calorimeter with a half width of $0.2 \lambda(\lambda-$ Nuclear interaction length, $\lambda_{\mathrm{Pb}}=18.0 \mathrm{~cm}$ used), cuts were applied which only included hits with $|x| \leq 3.6 \mathrm{~cm}$, $|y| \leq 3.6 \mathrm{~cm}$, and no cut on the $\mathrm{z}$ coordinate for the hits.

Results

Probably the most fundamental understanding we acquired about how

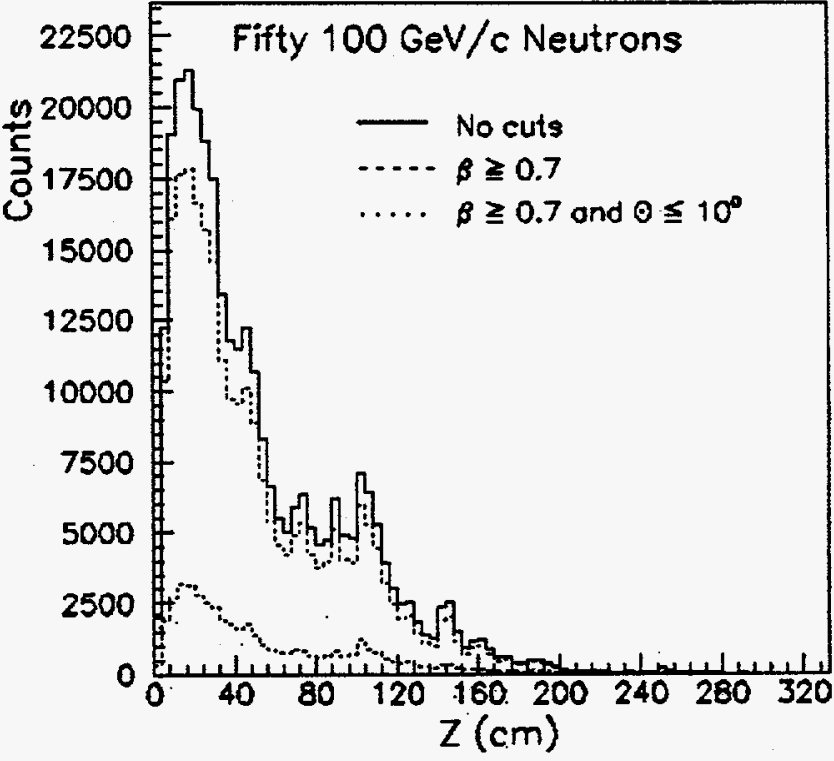

Figure 4. The number of charged particle hits per layer vs. distance in the calorimeter. Notice that the velocity cut has a relatively small effect on the number of hits whereas the angle cut has a large effect. 
Q-cal devices work was that it is the finite angle cut required to trap the Cherenkov photons in the fibers, and not the $\beta$ requirement, which limits the size of the showers. With hindsight this result makes sense. It's due to the fact that most of the hits in a $\mathrm{Pb}$ plastic scintillator sampling calorimeter are due to electrons and positrons. An electron or positron with kinetic energy as low as $\sim 200 \mathrm{keV}$ satisfies the $\beta$ threshold for Cherenkov radiation. This point is illustrated graphically in Fig. 4 which shows the number of hits versus depth in the Pb-plastic stack with no cuts applied to the hits, with the cut $\beta \geq 0.7$, and with the cuts $\beta \geq 0.7$ and $\Theta \leq 10$ degrees. The data in this plot was generated with the low energy cutoff values in GEANT given earlier. It is clear from the plot that the dominant reduction in the number of hits comes from the angle cut and not the velocity ( B) cut.

Due to the space constraints for a zero degree calorimeter at RHIC, discussed earlier, we studied the fraction of the energy lost, and the fluctuations in the energy deposited (i.e. Energy resolution) for various size calorimeters. This was accomplished, using PAW, by merely placing cuts on the $\mathrm{X}$ and $\mathrm{Y}$ coordinates of the hits which we included in our sums of hits. In all cases the cuts on $X$ and $Y$ were equal, which results in a square cross section for the calorimeter, and there was no cut placed on the $Z$ coordinate of the hits, and hence contributions from the entire $3 \mathrm{~m}$ length of the calorimeter were included.

Fig. 5 shows a plot of the half width of the calorimeter, in units of the nuclear interaction length for lead (18 $\mathrm{cm}$ used) versus the ratio of the energy deposited to the energy deposited in a calorimeter with a half width of 1 nuclear interaction length. This plot illustrates the point that the width of the showers which are detected in a Q-cal type calorimeter (with $\beta$ and $\Theta$ cut) are narrower than those in a standard sampling calorimeter. In particular, the plot shows that for our simplified simulation $770 \%$ of the shower is contained in a rectangular box with transverse dimensions of $3.6 \times 3.6 \mathrm{~cm}$ $(0.1 \lambda * 18 \mathrm{~cm} / \lambda * 2)$ for the Q-cal, whereas it takes transverse dimensions of about $7.2 \times 7.2 \mathrm{~cm}$ to contain about the same fraction of the shower in a standard calorimeter. Thus one can conclude that, based on this simulation, the effective shower size in a Q-cal type calorimeter is smaller than that in a standard sampling calorimeter.

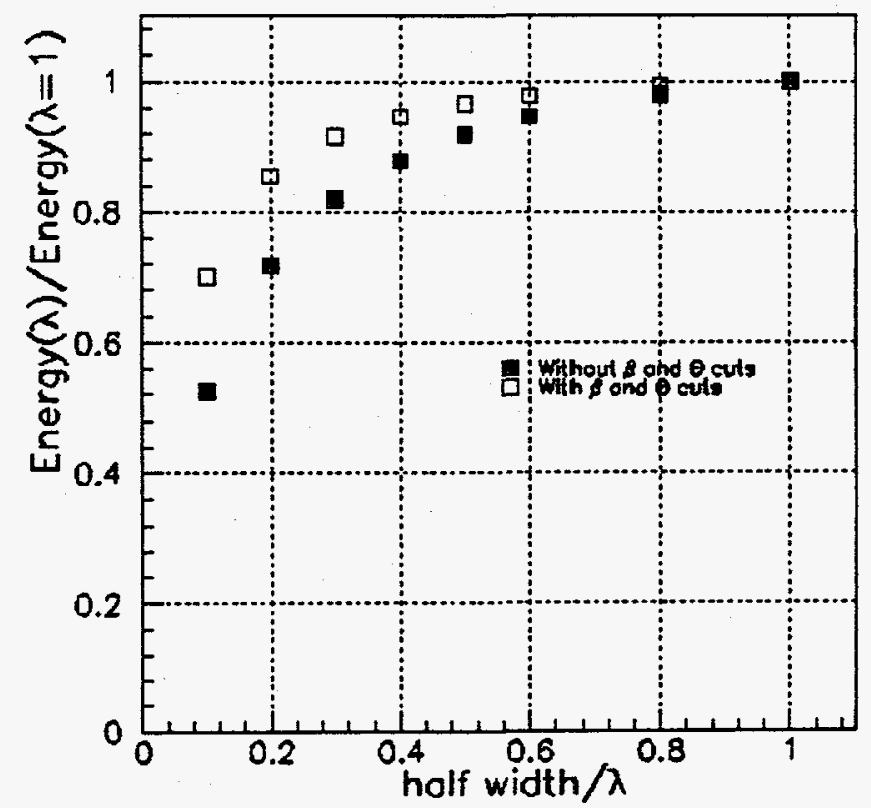

Figure 5. Plot of the half width of the calorimeter, in units of the nuclear interaction length for lead versus the ratio of the energy deposited to the energy deposited in a calorimeter with a half width of one interaction length. This plot illustrates the narrower effective shower size of the $Q$ cal type calorimeter. 
The next question which must be answered is 'what is the energy resolution', as a function of transverse size, for the two types of calorimeters. The analysis for the energy resolutions was done in the same manner as that for the shower widths. Cuts were placed on which hits were included in the various sums. The rms width of the sums were then divided by the means for the sums to arrive at the energy resolution. The results are shown in Fig. 6. There are a number of features in this plot which warrant mention. The first is that if one looks at the simulation results for the larger transverse size calorimeters (i.e. half width $/ \lambda \geq 0.5$ ) one sees that the standard calorimeter has the best resolution, followed by a Q-cal type

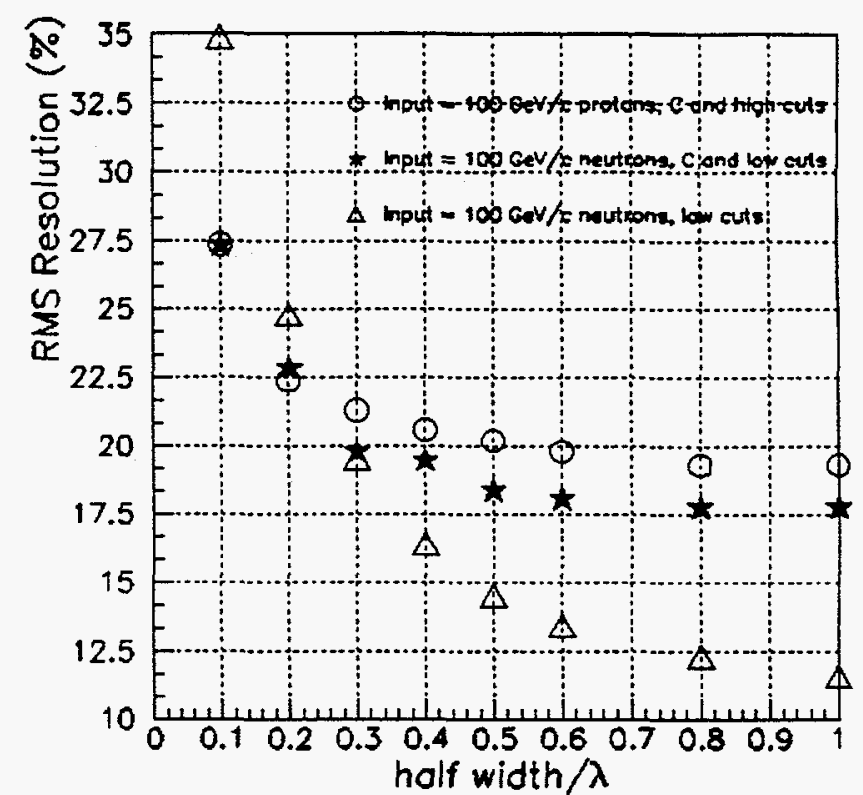

Figure 6. RMS energy resolution versus half width (in units of nuclear int. length) for calorimeters with square transverse cross sections. The $C$ in the legends refer to the $Q$-cal cuts ( $\beta \geq 0.7$ and $\theta \leq 10$ degrees). Please see text for definition of high and low cuts.

with low cutoff energies in GEANT, and finally by a Q-cal type with high cutoff energies in GEANT. This is due to the fact that as soon as the showers are fairly well contained in the calorimeter, the larger the number of hits the smaller is the statistical fluctuations in the number of hits. The point at which the Q-cal starts to give better resolution appears to be at a half width of about $0.25 \lambda( - \pm 4.5 \mathrm{~cm}$ in $\mathrm{x}$ and $\mathrm{y})$. This does not mean that for particles incident within $\sim 4.5 \mathrm{~cm}$ of an edge of a large calorimeter that one will have better energy resolution with a Q-cal type calorimeter. Due to the fact that the part of the shower away from the edge will be completely contained in the calorimeter, the point where one starts to get better resolution with a Q-cal will be somewhere closer toward the edge than the $4.5 \mathrm{~cm}$. To find this cross over point will require a re analysis of the data with different cuts.

\section{Q-cal simulations with more sophisticated fiber response}

The more precise Q-cal simulations were performed by slightly changing one of the standard Q-cal programs.

The dimensions of the detector were $1 \times 1 \times 3 \mathrm{~m}$ in the $\mathrm{X}, \mathrm{Y}$ and $\mathrm{Z}$ directions, respectively. It had a form of a parallelepiped with a 45 degrees tilt with respect to the beam (positive $Z$ ) axis. This tilt was chosen so that fibers point along the Cherenkov cone maximizing the probability of capturing the created photons. The absorber consisted of 4 $\mathrm{mm}$ thick lead sheets. The sheets were grooved and between them $1 \mathrm{~mm}$ thick fibers were positioned. The fibers were distributed in layers, touching each other laterally and creating layers similar to the plastic scintillator plates used for the simulations of the standard calorimeter. This particular distribution was not chosen only in order to better mimic the geometry of the standard calorimeter. It was found, both through simulations and experiment, that the lateral size of the showers is so small that even a small lateral 
distance between fibers can change the performance of the calorimeter. On the other hand, the thickness of the absorber plates was small when compared with the dimensions usually applied in Q-cal simulations. The distance between fiber layers along the direction of the incoming particles has a weaker influence on the calorimeter resolution, and increasing the thickness of the plates leads to a cheaper (less fibers) and more compensating calorimeter (through suppression of the response to the e.m. component of hadron showers). In order to decrease the influence of the early phases of hadronic shower development, the subsequent layers of fibers were laterally shifted by $0.5 \mathrm{~mm}$. In this way, even incident charged hadrons couldn't pass through multiple fiber layers without giving a response.

The simulations were performed using the standard GEANT simulation package, version 3.15. The energy cuts for all the tracked particles were defined by the energy corresponding to the threshold of Cherenkov effect and were equal to the "low cuts" used for simulations of the standard calorimeter in the previous section. The minimal steps were calculated by GEANT itself through the AUTO data card and were equal to 0.35 $\mathrm{mm}$ in lead and $0.22 \mathrm{~mm}$ in quartz.

The logic of the program was the following: particles from the shower in the detector were tracked through the standard GEANT procedure and when a charged particle hit a fiber, the response was read from an independently produced table. The light yield tables were produced by using simple geometrical considerations explained in Ref. 1. This approach was based on the fact that detailed simulations of trajectories of individual Cherenkov photons, although possible, would considerably increase the CPU time. As the Q-cal was conceived for work in the TeV energy range, the simple tracking of the showers takes considerable time even with relatively high energy cuts ( about $0.3 \mathrm{~s} / \mathrm{GeV}$ on Hewlett-Packard series 700 , model 735 ). Two different sets of data tables were used for these simulations. One set for fibers with a numerical aperture (NA) of 0.22 (doped silica clad fibers, the best with respect to the radiation resistance and transparency) and one for 0.37 (plastic clad fibers, with acceptable behavior up to the doses of the order of 0.6-0.7 Grad). The light yield calculations took into account the wavelength dependence and the quantum efficiency of a commercially available Philips XP 2020 Q photo cathode, so the elements of the table were the mean numbers of photoelectrons produced in this photomultiplier for every combination of pertinent parameters. As relevant parameters we took the particle velocity beta $=\mathrm{v} / \mathrm{c}$, the angle alpha and the distance $b$ between the particle trajectory and the fiber axis. For each charged particle crossing the fiber these parameters were calculated and a corresponding value taken from the tables. As the number of the produced photoelectrons is rather low (of the order of 1-2 per crossing), the influence of the poor photo statistics had to be taken into account. This was achieved by random generation of the number of "detected" photoelectrons according to a Poisson distribution. The non-integer number taken from the tables was used as the input value (mean of the Poisson distribution) for the POISSN randorn number generator from CERN MATHLIB package. As there are different ways of treating the produced photons, it should be noted that in this case we assumed: a) that all the photons exiting the fiber hit the photo cathode and b) that there is no particular material ensuring the optical contact between the fiber and the photo cathode. This means that a certain small part of the captured light will be reflected back from the front surface of the fiber. 

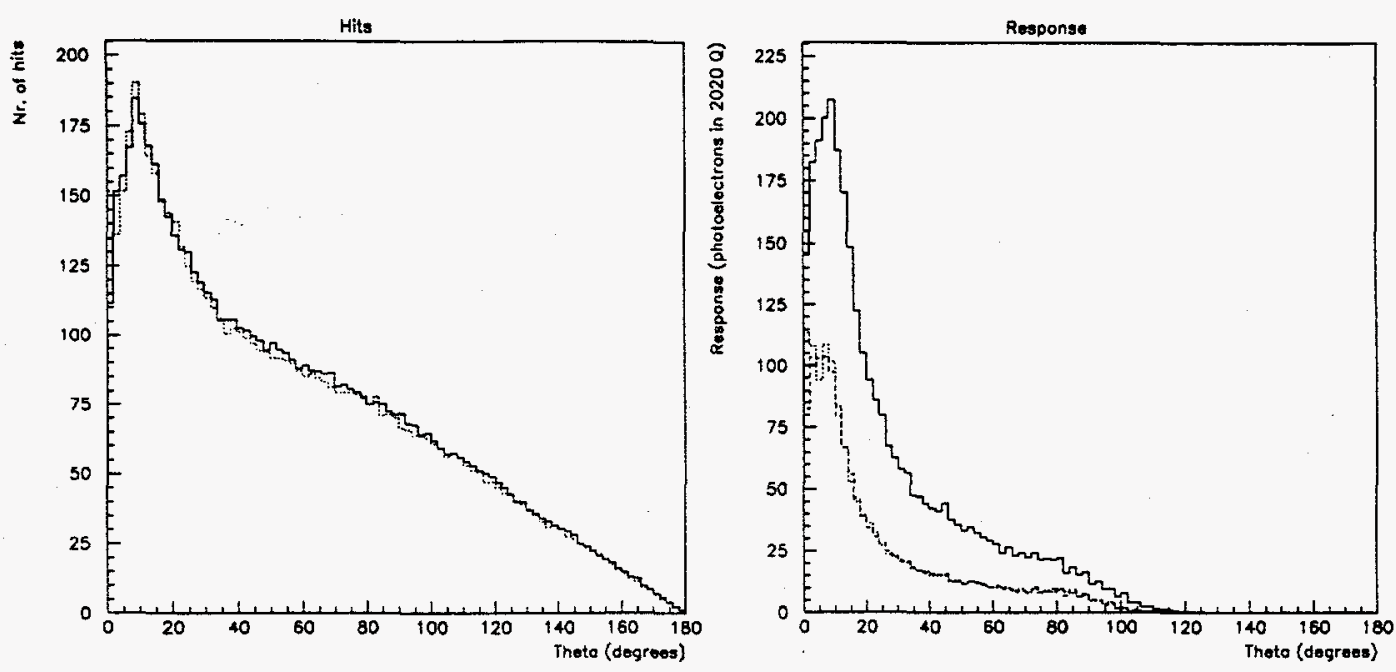

Fig. 7. Distribution of hits(a) and response (b) of the quartz calorimeter as a function of the angle wrt the direction of the incoming particles.

The obtained response was further processed in order to derive the shower size: the coordinates of the point of impact of the charged particle were used to define in which volume the photo electrons are created. The volumes had square cross sections in the X-Y plane, they were centered on the position of the initial particle, and their sides progressively increased from 1 to $100 \mathrm{~cm}$. The fluctuations of the longitudinal dimensions of the shower were not studied this time, so all the volumes had the length equal $3 \mathrm{~m}$. For each event, i.e. for each projectile, the response in different volumes was recorded in a ntuple and the analysis of the fluctuations was done at the end of the program, after the tracking was over. This ntuple and three summary histograms of the distributions of mean value, dispersion and resolution as functions of the sizes of fictive volumes were accompanied by the standard output of Q-cal simulations consisting of one ntuple with ten elements and forty nine twodimensional histograms. Three different sets of simulations were performed, each of them consisting of 100 simulated events. $100 \mathrm{GeV}$ neutrons were used as projectiles for two simulations with fibers having $\mathrm{NA}=0.22$ and

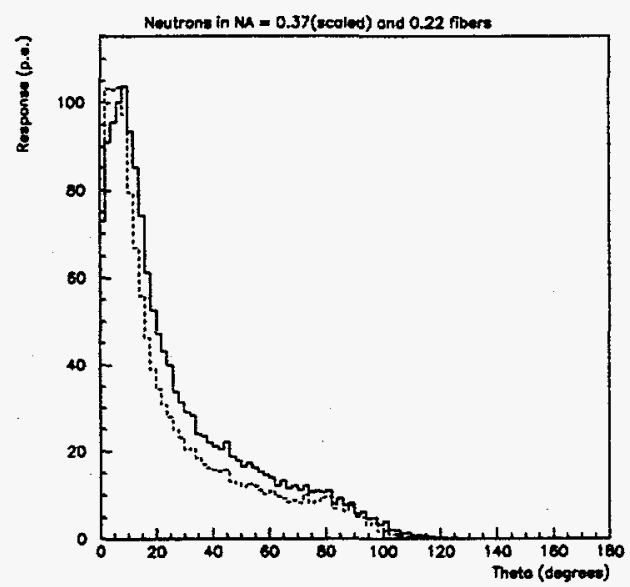

0.37 . For the fibers with $N A=0.22$ protons were also used as projectiles in order to check if there is a difference between the responses and shower sizes.

Some of the distributions taken by default are shown in Figs. 7a and b. They show the distributions of the number of hits by charged particles and the produced 
response as a function of the angle between the particle momentum and the beam axis for fibers with numerical apertures equal 0.22 and 0.37 . We see that, while the hits have a much more "isotropic" distribution, coming from the scattered electrons and positrons, the angular distribution of the response has a very clearly defined peak with full-width at half-maximum equal $\sim 20$ degrees. Obviously, the fibers act as "angular filters", sensitive only to the very core of the shower. The tail towards higher values of the angles comes from the fact that the angle between a fiber axis and the particle trajectory is not a function of only the angle theta, but also of the azimuthal angle phi, so even for quite high values of theta a "good" value for photon production can be achieved for some special values of phi. This is a manifestation of one of the basic properties of Cherenkov calorimetry - that the effects have always to be considered in three dimensional space. These two figures have some more information to offer: first, there is no particular difference in the angular

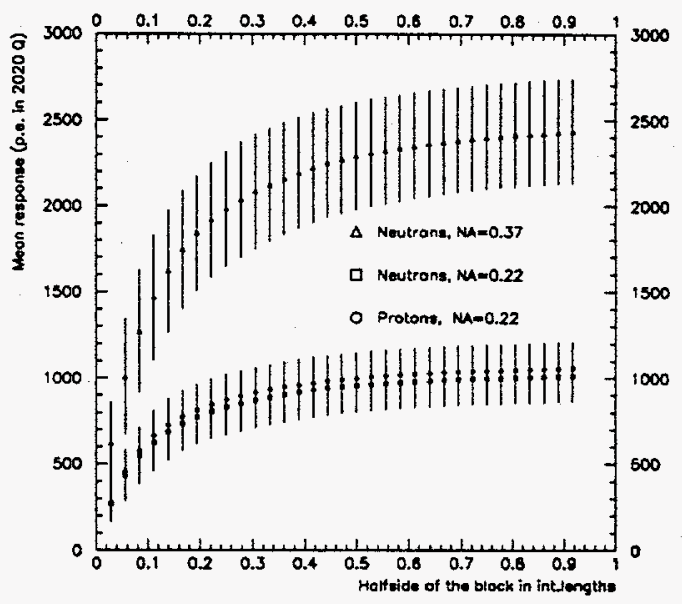
distribution of the hits for protons and neutrons. Second, the difference in responses for fibers with numerical apertures equal 0.22 and 0.37 is more in the amplitude (factor two) than in shape. Although we would expect significant broadening of the distribution, the difference at half maximum is only 2-3 degrees, as can be seen from Fig. 7c. The solid line corresponds to the distribution for fibers with $\mathrm{NA}=0.37$ divided by two, and the dotted line to the original distribution of the response for the fibers with $N A=0.22$.

The distributions of the response as a function of the size of the calorimeter are shown in Fig. 8. The data points give mean values and error bars give the (rms.) dispersion. We see that the response to protons and neutrons is for all practical purposes equal. A much more important feature is the difference between the curves for NA $=0.37$ and NA $=0.22$ fibers, actually the lack of any difference. When the response curves are normalized to their maximal values the shower containment is practically the same for the two different types of fibers. The diagrams are shown in Fig. 9. This result is in agreement with the effect seen in Fig. 7c: that the relatively small capturing angle of the fibers becomes

Figure 8. Mean response of the calorimeter as a function of its size. The error bars correspond to dispersion of the response for a given size. "smeared" due to the shower spread.

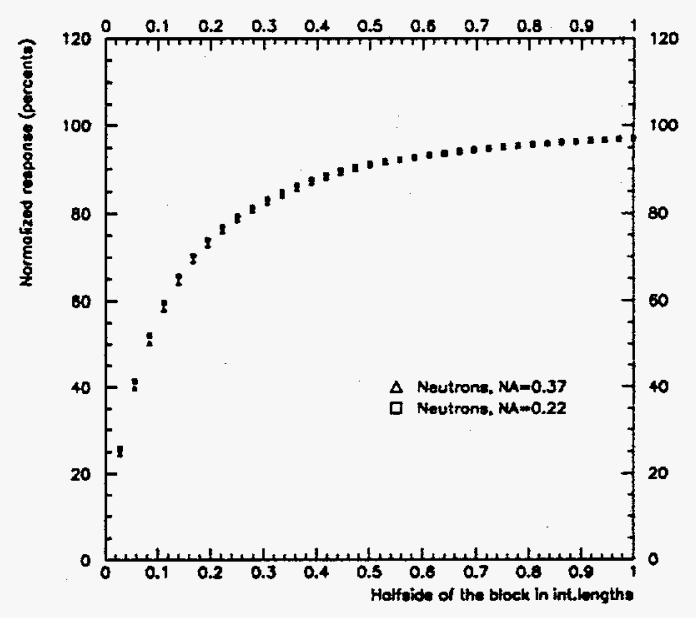

Figure 9. Comparison of normalized responses to $100 \mathrm{GeV}$ Neutrons for fibers with NA $=0.22$ and NA $=0.37$ as a function of the calorimeter size. 
However, the resolution is not the same, as can be seen from the Fig. 10. The fibers with higher numerical aperture have better light collection and give a better resolution. This is an encouraging result from the point of view of the resolution of the calorimeter: with higher NA fibers we can achieve better resolution within the same volume. The development of new radiation resistant cladding materials is a part of the Q-cal collaboration program. The rad hard fibers with higher NA could further improve the characteristics of the detector. Of course, this effect should be experimentally checked first, and then, if possible an upper limit for the numerical aperture of the fibers found. This limit should correspond to the value when the observed shower shape starts to visibly grow.

The slight difference in resolution for protons and neutrons was traced to the presence of three high energy muons in the data sample for neutrons, traveling almost in the direction of the incident beam and therefore producing the maximum light. This means that, in order to make more detailed and reliable studies of the resolution, we need to work with a much larger number of events. The fact that incident protons produce light even before the first interaction can be another explanation for the better resolution for protons than neutrons for very small detector volumes: while the presence of the muons is a stochastic and not very probable event, especially in the correct angular interval, the charged projectiles are always producing the light and the fluctuations are determined by the depth of the first interaction.

In any case, although the obtained values for the resolution should be taken with caution, the calculated shower sizes agree in principle with measured values for

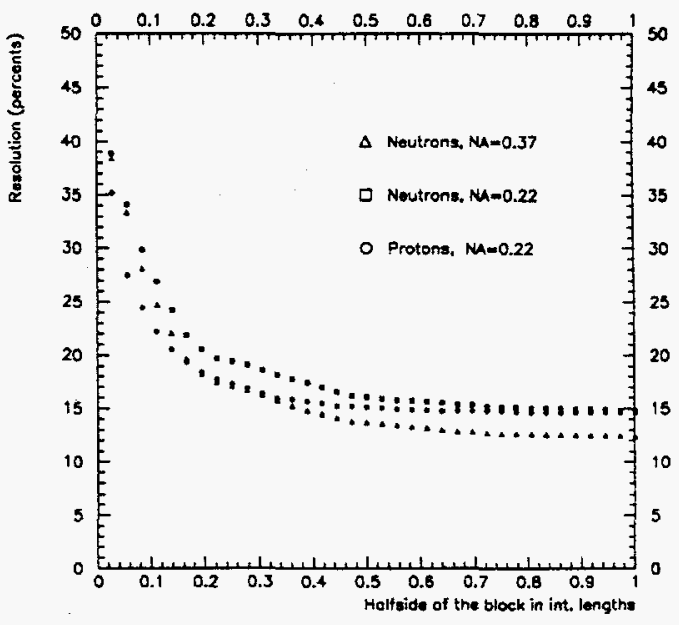

Figure 10. Resolution of the calorimeter as a function of the calorimeter size. $160 \mathrm{GeV}$ pions shown in Fig. 11 The figure shows the comparison of shower profiles detected in SPACAL and Q-cal. The data points for Q-cal were derived from the fit to the (experimental) edge scan, while the data for SPACAL ${ }^{2}$ were calculated from a 
parameterization of the radial shower sizes given in ref. 2. Both curves are based on experimental results and the advantage of Q-cal is obvious. However, these numbers cannot be used directly for the case of RHIC as neither energies nor projectiles are the same.

\section{Conclusions}

We feel that instrumenting the very forward direction with calorimetry near the beam tube can offer a useful way to characterize heavy ion collisions at RHIC.

Because of space limitations such a calorimeter would be limited in transverse dimension. Furthermore, many of the hadrons of interest will impact the calorimeter near its edge. We therefore find it of interest to examine calorimeter sampling techniques which are intrinsically less sensitive to shower products which are at a larger distance from the core.

We simulated the Q-cal calorimeter in which Cherenkov light is emitted in Quartz fiber ribbons and then transported by total internal reflection to the PMT. First a simplified simulation was carried out in which we characterize this sampling technique as a filter on velocity and angle of shower products. Comparison with unfiltered (scintillator) sampling indeed shows a decreased variation in response due to lateral containment effects.

A second simulation based on realistic simulation of the Quartz fiber system confirms the characteristics inferred from the simplified model.

Before proceeding further on the specifics of a calorimeter design for RHIC, we feel that further work should be done using new data from the AGS and CERN to better understand the physics of zero degree event characterization. In particular we would like to know whether protons could equally well be used for event characterization. In this case the space restrictions would be much less severe.

We wish to thank Edward Kistenev and Dan Weiss for useful discussions.

\footnotetext{
'Thesis of D. Lazic, Centre de Recherches Nucleaires, Strasbourg, CRN 93-38, N. d'ordre 1534, 1993.

${ }^{2}$ D. Acosta et al. NIM A316 (1992) 184-201.
}

\section{DISCLAIMER}

This report was prepared as an account of work sponsored by an agency of the United States Government. Neither the United States Government nor any agency thereof, nor any of their employees, makes any warranty, express or implied, or assumes any legal liability or responsibility for the accuracy, completeness, or usefulness of any information, apparatus, product, or bility for the accuracy, complets that its use would not infringe privately owned rights. Reference herein to any specific commercial product, process, or service by trade name, trademark, manufacturer, or otherwise does not necessarily constitute or imply its endorsement, recommendation, or favoring by the United States Government or any agency thereof. The views and opinions of authors expressed herein do not necessarily state or reflect those of the United States Government or any agency thereof. 
